\title{
Computational Model for Microbubble Enhanced Performance of Airlift Bioreactor (ALB)
}

\author{
W. C. $\mathrm{Ng}^{1}, \mathrm{~S}^{\text {Brittle }}{ }^{2}$, R. Howell ${ }^{1}$, W. B. Zimmerman ${ }^{2}$ \\ ${ }^{1}$ Department of Mechanical Engineering, University of Sheffield. \\ ${ }^{2}$ Department of Chemical and Biological Engineering, University of Sheffield.
}

\begin{abstract}
This paper presents a computational model for microbubble enhanced performance of an airlift bioreactor (ALB). Five different bubble diameters were defined in the model under the same conditions ( $440 \mu \mathrm{m}$ to $1 \mathrm{~mm}$ bubble diameter). The computational model parameters and the size of the ALB were defined by referring to experimental work done previously. The main objective of the model is to study the effect of bubble size on the rising velocity and the liquid flow velocity in the airlift reactor (ALB). The results obtained from the computational model shows that microbubbles have a better performance over larger bubbles because microbubbles have better gas hold up due to slow rise velocity and are able to increase the flow velocity due to their high surface area to volume ratio.
\end{abstract}

Keywords Airlift Bioreactor; Bubble Rise Velocity; Comsol; Liquid Flow Velocity; Microbubbles

\section{INTRODUCTION}

The characteristics of ALBs have been studied and appear to increase mixing throughout the reactor and reduce the bubble coalescence compared to the bubble column and stirred tank bioreactor. Nevertheless, there are still disadvantages such as high energy requirement due to the tall design leading to high gas hold-ups, long bubble residence times, a region of high hydrostatic pressure near the aerator at the base of the fermenter, and cell damage due to bubble bursting particularly with animal cell culture. The hydrodynamic within the ALB is an important factor that affect the performance of the ALB. Therefore, there are many improvement and optimisation studies of the ALB. However, most of the previously published studies have aimed to improve the ALB performance by modifying the parameter of the ALB [1].

Recently, microbubbles have been shown to effectively enhance performance of several applications, including waste water aeration, algal growth, carbon capture, separation processes for yeast and algae, due to the surface area to volume ratio and low rise velocity. These advantages of microbubbles, along with high gas hold up, high liquid circulation velocity and liquid mixing time coincidently happen to be the disadvantages of the ALB. It is strongly believed that utilising microbubbles will improve the performance of the ALB. As a result, this computational model studies the behaviour of micro sized bubbles in the ALB.

\section{COMPUtATIONAL MODELLING OF ALB}

\subsection{LAMINAR BUBBLY FLOW MODEL}

The laminar bubble flow interface of Comsol Multiphysics was applied to setup a multiphase flow model for gas bubble rising through a liquid. The laminar bubbly flow model solves for the liquid: velocity, pressure and volume fraction of the gas phase. The momentum transport equation was applied for the mixture.

In general, buoyancy causes the bubbles to rise through a liquid. It can be assumed that the pressure forces approximately balance the viscous drag forces on a gas bubble. Therefore, the pressure drag balance slip velocity model was defined. In addition, the bubble diameters defined in the computational model are smaller than $2 \mathrm{~mm}$ and a Hadamard-Rybczynski drag coefficient model was applied. The Hadamard-Rybczynski drag law is for spherical gas bubbles smaller than $2 \mathrm{~mm}$ in liquids.

$$
C_{d}=\frac{16}{R e_{b}}, \quad R e_{b}=\frac{d_{b} \rho_{l}\left|u_{\text {slip }}\right|}{\mu_{l}}
$$

where $C_{d}$ is drag coefficient, $\operatorname{Re}_{b}$ is bubble Reynolds number, $d_{b}$ is bubble diameter, $u_{\text {slip }}$ is slip velocity, and $\rho_{l} \& \mu_{l}$ is the liquid density and viscosity respectively [2].

\subsection{BOUNDARY CONDITIONS (BCS)}

The BCs on the reactor walls and draft tube was defined as no slip BCs for the liquid phase while "no gas flux" BCs were used for the gas phase.

$$
u_{l}=0, \quad n\left(\varnothing_{g} u_{g}\right)=0
$$

The BCs for the aerator bubbling surface was defined as no slip BCs for liquid with "gas flux" BCs for gas.

$$
-n\left(\tilde{\rho}_{g} u_{g}\right)=N_{\emptyset_{a} \rho_{a}}
$$

The top liquid surface was defined as "Gas outlet" BC. These $B C s$ imply gas leaving the reactor at $U_{g}$ with no other constraints at the boundary. For the liquid phase, a slip boundary condition were used.

$$
u_{l} n=0
$$

where $u_{l}$ is liquid phase velocity, $u_{g}$ is gas phase velocity, $\tilde{\rho}_{g}$ is effective gas phase density, and $\emptyset_{g}$ is gas volume fraction. 


\section{RESULTS}

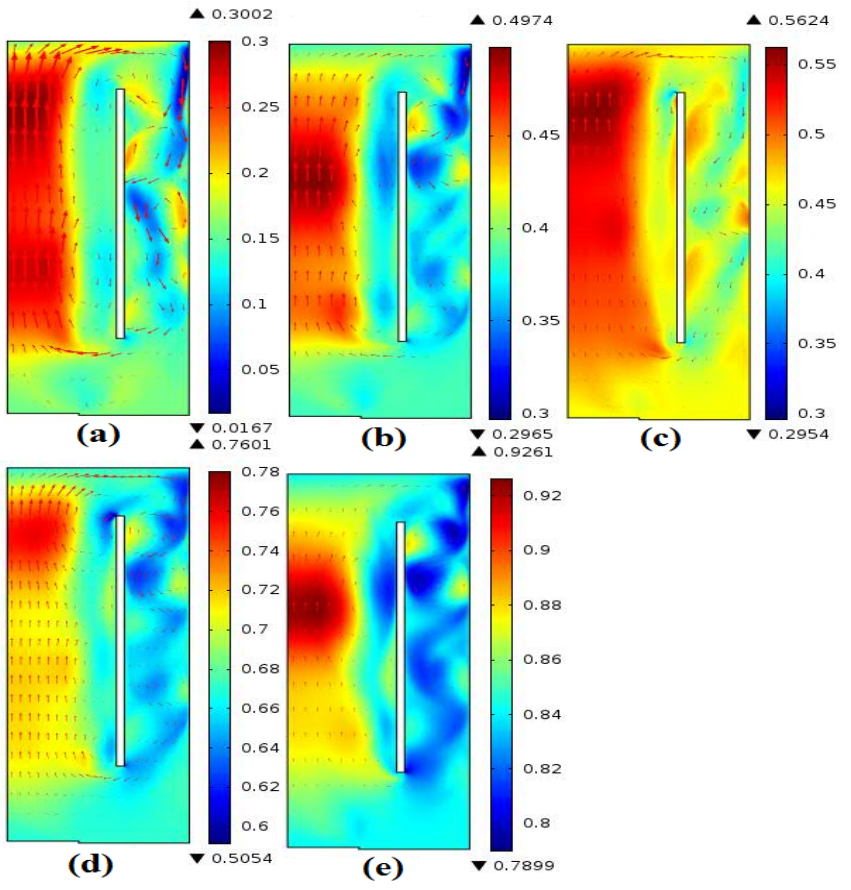

Figure 1(a). Contour plot and vector plot of bubble rise velocity of $440 \mu \mathrm{m}$; (b). $690 \mu \mathrm{m}$; (c). $755 \mu \mathrm{m}$; (d). $910 \mu \mathrm{m}$; (e). $1 \mathrm{~mm}$ diameter bubbles.

Fig. 1(a-e) shows the gas phase contour plot of bubble rise velocity of 5 bubble diameters at the same flow rate. The overall results indicate the bubble rise velocity increases with the bubble diameter at the same inlet flow rate.

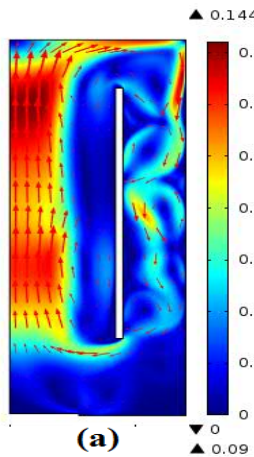

0.144 $\Delta 0.1115$

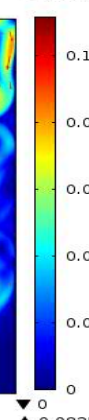

(b)

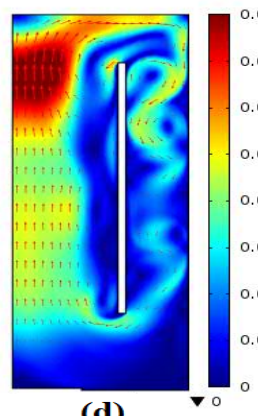

(d)

igure 2(a). Contour plot and vector plot of flow velocity of $440 \mu \mathrm{m}$; (b). 690 $\mu \mathrm{m}$; (c). $755 \mu \mathrm{m}$; (d). $910 \mu \mathrm{m}$; (e). $1 \mathrm{~mm}$ diameter bubbles.
Fig. 2 (a-e) shows the liquid phase contour plot driven by 5 bubble diameters at the same flow rate. It can be observed that microbubbles cause significantly higher liquid circulation compared with the large bubbles at the same
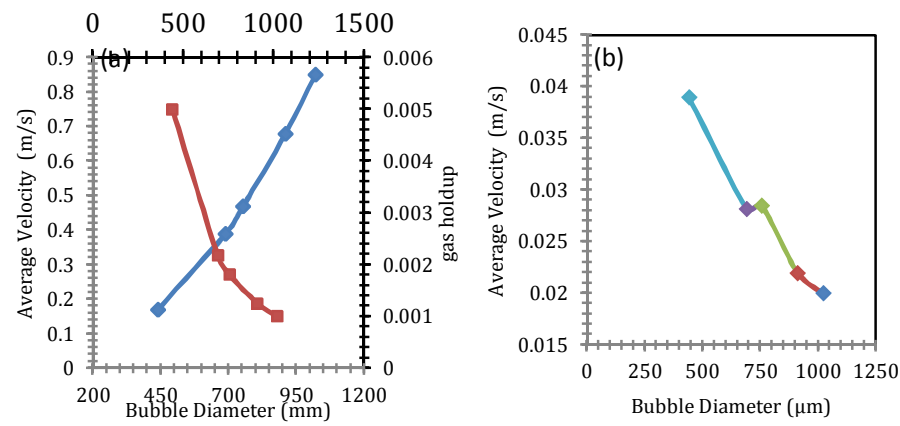

inlet flow rate.

Figure 3(a). Average bubble rise velocity (blue) and gas holdup (red) as a function of bubble diameter; (b). Average flow velocity as a function of bubble diameter.

Fig. 3(a) \& (b) show the average velocities of 5 different bubble diameter, calculated by averaging 30 sets of data at 9 positions in the ALB. Fig. 3(a) illustrates the relation between the bubble diameter, the gas holdup due and the bubble rise velocity. A smaller bubble diameter with the low average rise velocity leads to the higher gas holdup due to a longer residence time in the liquid. Fig. 3(b) illustrates how microbubbles cause higher liquid circulation due to the high surface area to volume ratio compare with the large bubble at the same inlet flow rate in the ALB.

\section{CONCLUSIONS}

The characteristics of the microbubbles were determined and summarised as below:

i. The bubble rising velocity increases with the bubble diameter in the ALB. Therefore, microbubbles lead to a better gas hold up due to the longer residence time in the water.

ii. Smaller bubble sizes are able to increase the flow velocity which also helps in the suspension of biological matter and improve mixing.

iii. Controlling bubble size can be an important factor that affects the performance of the ALB.

\section{ACKNOWLEDGEMENTS}

We acknowledge support for the study of microbubble dynamics from the EPSRC.

\section{REFERENCES}

1. K.ZhenYing, D.J. Gilmour, Y.Zhen Shi, W. B. Zimmerman, Growth Enhancement of Dunaliella Salina by Microbubble induced Airlift Bioreactor(ALB)- The Relation Between Mass Transfer and Growth Rate,2013.

2. Mahmood K. H, H. C. H. Bandulasena, W. B. Zimmerman, $\mathrm{CO} 2$ Mass Transfer Induced through an Airlift Loop by a Microbubble Cloud Generated by Fluidic Oscillator, 2011. 